\title{
A phase III, multicentric, open-label, two-arm, parallel group, active-control, randomized, comparative clinical study to evaluate efficacy and safety of RituxiRel ${ }^{\mathrm{TM}}$ arm (rituximab) with reference arm (rituximab) in patients with non-Hodgkin's lymphoma
}

\begin{abstract}
Introduction: Non-Hodgkin's lymphoma (NHL) is the sixth most common hematological malignancy in adults, with B-cell lymphomas accounting for $85 \%$ of all NHLs. Diffuse large B-cell lymphoma (DLBCL) is the most common subtype of NHL and follicular lymphoma (FL) is the second most common form of B-cell NHL.

Materials and Methods: The primary objective of this study is to assess the efficacy of Rituxire ${ }^{\mathrm{TM}}$ arm with reference arm, whereas the secondary objective is to evaluate safety of Rituxire ${ }^{\mathrm{TM}}$ arm with the reference arm in patients diagnosed with NHL.

Results: The first patient was enrolled on April 30, 2012 and the efficacy and safety analysis was performed at 24 weeks. The objective response rate (ORR) was observed to be $87.87 \%$ in Rituxirel ${ }^{\mathrm{TM}}$ arm. $45.45 \%$ patients showed complete response and $42.42 \%$ patients showed partial response in Rituxirel ${ }^{\mathrm{TM}}$ arm. The ORR was observed to be $86.66 \%$ in the reference arm. $33.33 \%$ patients showed complete response and $53.33 \%$ patients showed partial response in reference arm in the Rituxirel ${ }^{\mathrm{TM}}$ arm, the most commonly reported treatment-emergent adverse events (TEAEs) related to blood and lymphatic system disorders were $52.94 \%$, whereas in the reference arm, the reported TEAEs related to blood and lymphatic system disorders were $70 \%$.

Conclusion: Based on the results from the efficacy and safety analysis at week 24, RituxirelTM arm was found to be as effective and safe as the reference arm. Rituxire $\mathrm{I}^{\mathrm{TM}}$ arm can be a prudent option to the reference arm, in patients undergoing treatment for DLBCL or FL.
\end{abstract}

Keywords: Comparative efficacy and safety, diffuse large B-cell lymphoma, follicular lymphoma, rituximab, survival

\section{INTRODUCTION}

Diffuse large B-cell lymphoma (DLBCL) is the most common subtype of non-Hodgkin's lymphoma (NHL), whereas follicular lymphoma $(\mathrm{FL})$ is the second most common form of B-cell NHL. ${ }^{[1]}$ Survival can be improved by $10 \%-20 \%$ with strategies such as use of rituximab and dose intensification of the $\mathrm{CHOP}$ regimen. ${ }^{[2]}$ Rituximab induces a rapid depletion of normal CD20-expressing B-cells in the peripheral blood. ${ }^{[3]}$

Rituximab is produced by recombinant DNA technology; Rituxirel $^{\mathrm{TM}}$ is from Reliance Life Sciences (RLS). The purpose

\begin{tabular}{|l|c|}
\hline \multicolumn{2}{|c|}{ Access this article online } \\
\hline \multirow{2}{*}{$\begin{array}{l}\text { Website: } \\
\text { www.asjo.in }\end{array}$} & Quick Response Code \\
\hline \multirow{2}{*}{$\begin{array}{l}\text { DOI: } \\
\text { 10.4103/ASJO.ASJO_29_16 }\end{array}$} & \\
& \\
\hline
\end{tabular}

of the study was to establish biosimilarity in terms of efficacy and safety between Rituxire $\mathrm{I}^{\mathrm{TM}}$ arm and the reference

\section{Prasad Dattatray Apsangikar, Sunil Chaudhry, Manoj Murlidhar Natk, Parvez Kozgi}

Medical Affairs Group, Clinical Research Group, Reliance Life Sciences, Rabale, Navi Mumbai, Maharashtra, India

Address for correspondence: Dr. Prasad Dattatray Apsangikar, Medical Affairs Group, Reliance Life Sciences, Rabale - 400 701, Navi Mumbai, Maharashtra, India.

E-mail: prasad.apsangikar@relbio.com

This is an open access article distributed under the terms of the Creative Commons Attribution-NonCommercial-ShareAlike 3.0 License, which allows others to remix, tweak, and build upon the work non-commercially, as long as the author is credited and the new creations are licensed under the identical terms.

For reprints contact: reprints@medknow.com

How to cite this article: Apsangikar PD, Chaudhry S, Naik MM, Kozgi P. A phase III, multicentric, open-label, two-arm, parallel group, active-control, randomized, comparative clinical study to evaluate efficacy and safety of RituxiRel ${ }^{\mathrm{TM}}$ arm (rituximab) with reference arm (rituximab) in patients with non-Hodgkin's Iymphoma. Asian J Oncol 2017;3:17-22. 
arm at end of 24 weeks with the first patient enrolled on April 30, 2012.

\section{MATERIALS AND METHODS}

This was a prospective, multicentric, open-label, two-arm, parallel-group, active-control, randomized (4:1) comparative clinical study, which was ongoing at 19 sites in India, between 2012 and 2014 with assessment for primary endpoints at 24 weeks and with a provision to follow-up till 5 years to observe overall survival (OS) rate. ${ }^{[4]}$

The study was designed and conducted with the principles of good clinical practice, with applicable regulatory requirements. This study was approved by the Institutional Ethics Committee at each site and eligible patients provided written informed consent before participating in the study. The study evaluated the comparative efficacy and safety of Rituxirel $^{\mathrm{TM}}$ and the reference arm in patients with NHL.

\section{Study design}

A total of 105 patients were enrolled in the study across the centers, in two arms (i.e., Rituxirel ${ }^{\mathrm{TM}}$ and reference arm in 4:1 ratio). After randomization (4:1), approximately 84 patients were enrolled in Rituxirel ${ }^{\mathrm{TM}}$ arm (rituximab; RLS Pvt Ltd., Navi Mumbai) and 21 patients in reference arm (innovator rituximab). The sample size of the patient population was as per regulatory requirement.

The dose of Rituxirel ${ }^{\mathrm{TM}} /$ reference arm for NHL was $375 \mathrm{mg} / \mathrm{m}^{2}$ intravenous infusion on day 1 of each cycle (21 days) for six cycles in combination with chemotherapy (CHOP) during induction phase. The maintenance dose was given as per the investigator's discretion, for respective condition/indication.

All patients were assessed for objective response rate (ORR) (complete response and partial response) as assessed by RECIST 1.1 criteria at week 24, disease progression-free survival at 2 years, and $O S$ rate at 5 years.

The pharmacodynamic parameter (absolute B-cell count in the peripheral blood) was assessed in 42 patients in $1: 1$ ratio (21 patients in each arm: first 21 patients of Rituxirel ${ }^{\mathrm{TM}}$ arm and remaining 21 patients of reference arm). Blood samples were collected at day 1 ( $0 \mathrm{~h}$ : within $1 \mathrm{~h}$ before drug administration and at $12 \mathrm{~h}$ after drug administration), day 2 ( $24 \mathrm{~h})$, day 3 (48 h), day 4 (72 h), day 7 , day 14 and at week 4 , week 7 , week 10 , week 13 , week 16 , week 24 , and 2 years before drug administration.

\section{Patient population}

One hundred and five patients were enrolled in the trial who had DLBCL or follicular B-cell NHL. Patients between 18 years and 65 years and diagnosed with CD20 positive, patients with at least one target lesion lymph node with short axis $\geq 15 \mathrm{~mm}$ as shown by computed tomography scan with contrast, Eastern Cooperative Oncology Group performance status 0-2 and life expectancy with more than 6 months were enrolled in the study. Patients with active transformed lymphoma or history of central nervous system (CNS) disease (either CNS lymphoma or lymphomatous meningitis), concomitant malignancy, serum creatinine $>2.0$ times of upper normal limit, alkaline phosphatase $\geq 1.5$ times of upper normal limit, platelet count $<100,000 / \mathrm{mcg} / \mathrm{L}$, hemoglobin $<8.0 \mathrm{~g} /$ $\mathrm{dL}$, absolute neutrophil count $<1.5 \times 10^{9} / \mathrm{L}$, and serum immunoglobulin $\mathrm{G}$ level $<3.0 \mathrm{~g} / \mathrm{L}$ were excluded from the trial; history of prior chemotherapy, stem cell transplant and radiotherapy; patients with a history of high dose, systemic, steroid therapy within 6 weeks, previous use of nonhuman monoclonal antibody therapy, known hypersensitive to murine proteins, and pregnant women were also excluded from the trial.

\section{Study treatment: Dose and dosing schedule}

As per randomization (4:1), approximately 84 patients were administered Rituxirel $^{\mathrm{TM}}$ and 21 patients were administered the reference arm along with $\mathrm{CHOP}$ regimen. The dose of Rituxirel ${ }^{\mathrm{TM}} /$ reference arm administered was $375 \mathrm{mg} / \mathrm{m}^{2}$ intravenous infusion on day 1 of each cycle (21 days) for six cycles in combination with chemotherapy (CHOP) during induction phase, i.e., R-CHOP treatment cycle. Concomitant medication allowed during the trial was paracetamol, chlorphenamine, ondansetron, and granulocyte-colony stimulating factor.

\section{Efficacy assessments}

Efficacy endpoints assessed were ORR (complete response and partial response) by RECIST 1.1 criteria at week 24 , proportion of patient with ORR (complete response and partial response) assessed by RECIST 1.1 criteria at 10 weeks, 24 weeks, 1 year, 1.5 years, and 2 years, and proportion of patients with stable disease (SD) and progressive disease (PD) at week 24. Change in absolute B-cell count in the peripheral blood after Rituxirel ${ }^{\mathrm{TM}} /$ reference arm administration at 24 weeks after first cycle compared to baseline..

Disease progression-free survival would be assessed from time of randomization to progression, relapse, or death from any cause at 2 years, OS rate at 5 years in this ongoing study.

\section{Safety assessments}

Safety assessments included recording of all adverse events (AE) and serious $\mathrm{AE}$ (SAE) from time of enrollment of patient 
till the end of study in both the arms. Treatment-emergent adverse events (TEAEs) were followed till their resolution.

\section{Statistical analysis}

Comparative statistical analyses were performed using the $\mathrm{SAS}^{\circledast}$ (9.2 or higher version on windows) system for primary and secondary endpoint data. Descriptive statistics were presented for all efficacy and safety parameters across all visits.

\section{Analyses and data monitoring}

An analysis for the primary endpoints was done when all patients completed 24 weeks of the study. A follow-up analysis for the endpoints is planned to be performed when all patients complete 2 years of the study.

Primary analyses were performed by the intention-to-treat (ITT) method. As per ITT population, 86 patients were randomized in the Rituxirel ${ }^{\mathrm{TM}}$ arm and 22 patients were randomized to the reference arm. ITT population included all patients who were randomized in the study while safety population included all patients who were randomized and received at least one single dose of study medication. A total of 105 patients were included in the safety population, i.e., 85 patients in Rituxirel $^{\mathrm{TM}}$ arm and 20 patients in the reference arm.

Superiority was assessed by means of an one-tailed $t$-test comparing the mean treatment difference with zero. For other analyses, two-sample comparisons were performed with the use of Student's $t$-test or a Wilcoxon rank-sum test for continuous measures and a Chi-square test or Fisher's exact test for binary measures. Adjusted analyses were performed by the means of analysis of covariance or logistic regression. Rates of AEs were compared with the use of Poisson regression. All statistical tests were two-sided, and a $P<0.05$ was considered to indicate statistical significance.

\section{RESULTS}

Demographic and other baseline characteristics [Table 1] As per ITT population, 86 patients were randomized in Rituxirel $^{\mathrm{TM}}$ arm and 22 patients were randomized in reference arm.

Out of 86 patients randomized in the Rituxirel ${ }^{\mathrm{TM}} \mathrm{arm}$, 49 patients were male (56.98\%) and 37 (42.53\%) patients were female. The mean age of patients the randomized in the Rituxirel ${ }^{\mathrm{TM}}$ arm was 47.8 years.

Out of 22 patients randomized in the reference arm, $16(72.73 \%)$ patients were male and $6(28.57 \%)$ patients were female. The mean age of patients randomized in the reference arm was 52.7 years.

\section{Efficacy results}

The efficacy assessment at week 24 for Rituxirel ${ }^{\mathrm{TM}} /$ reference arm is as follows.

\section{Primary efficacy analysis}

The primary efficacy analysis was performed using per protocol (evaluable) population (101 patients). A total of 81 evaluable patients were considered for secondary efficacy analysis. A total of 66 evaluable patients were included from Rituxirel $^{\mathrm{TM}}$ arm and 15 from the reference arm.

The primary efficacy parameter, ORR, was evaluated using RECIST 1.1. The outcome of this analysis is as follows:

The object response rate was observed to be $87.87 \%$ in Rituxirel ${ }^{\mathrm{TM}}$ arm. $45.45 \%$ patients showed complete response and $42.42 \%$ patients showed partial response in Rituxirel ${ }^{\mathrm{TM}}$ arm. The ORR was observed to be $86.66 \%$ in the reference arm. 33.33\% patients showed complete response and $53.33 \%$ patients showed partial response in the reference arm [Figure 1]. The analysis of primary efficacy endpoint, i.e., ORR at week 24 shows comparable response for both Rituxirel ${ }^{\mathrm{TM}}$ and the reference arm $(87.87 \%$ vs. $86.66 \%$ ). The proportions of patients showing ORR in each arm were compared for statistical significance and the difference was found to be nonsignificant $(P=0.89656)$ [Figure 1].

\section{Secondary efficacy analysis}

Secondary efficacy assessments included at this stage of the study were proportion of patients with ORR assessed by RECIST 1.1 criteria at 10 weeks, proportion of patients with SD and PD at week 24.

Another secondary efficacy assessment included in this study was the pharmacodynamics response evaluated by change in absolute B-cell count in the peripheral blood after Rituxirel ${ }^{\mathrm{TM}}$ / reference arm administration at 24 weeks after the first cycle compared to baseline.

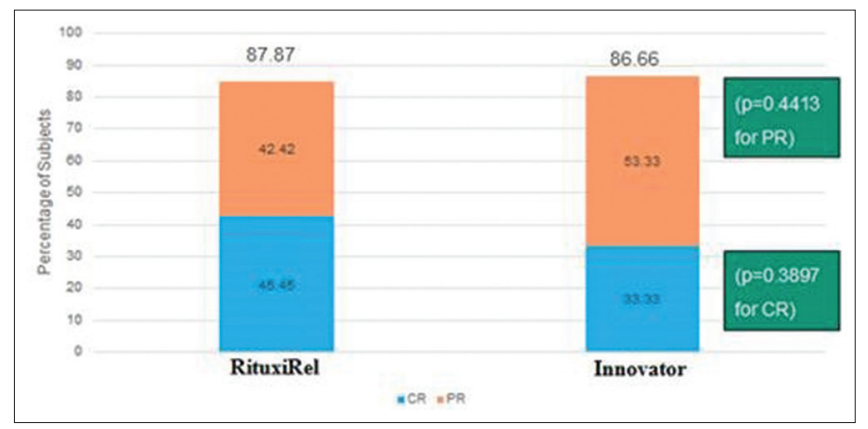

Figure 1: Objective response rate comparison: Rituxirel $^{\mathrm{TM}}$ and the reference arm at week 24 
The results of the secondary efficacy endpoint analysis were as follows:

The ORR was observed to be $81.71 \%$ in Rituxirel ${ }^{\mathrm{TM}}$ arm. $23.17 \%$ patients showed complete response and $58.54 \%$ patients showed partial response in Rituxirel ${ }^{\mathrm{TM}}$ arm at week 10 . The ORR was observed in $89.47 \%$ patients in reference arm. 5.26\% patients showed complete response and $84.21 \%$ patients showed partial response in reference arm at week 10 [Figure 2].

The proportion of nonresponders at week 24 was comparable; $8(9.76 \%)$ patients in Rituxirel ${ }^{\mathrm{TM}}$ arm and $2(10.52 \%)$ patients in reference arm.

\section{Pharmacodynamics evaluation}

The pharmacodynamics parameter (absolute B-cell count in the peripheral blood) assessment was done in 42 patients in $1: 1$ ratio (21 patients Rituxirel ${ }^{\mathrm{TM}}$ arm and 21 patients reference arm). Baseline B-cell assessment was done for total 20 patients who were administered Rituxirel ${ }^{\mathrm{TM}}$ and 19 patients who were administered reference arm. The pharmacodynamics assessment was done with change from baseline in absolute B-cell count in the peripheral blood after Rituxirel ${ }^{\mathrm{TM}} /$ reference arm administration at 4 weeks and 24 weeks. The baseline mean B-cell count observed for Rituxirel $^{\mathrm{TM}}$ arm was 520.4, which showed a decline after the start of treatment with Rituxirel ${ }^{\mathrm{TM}}$. At week 24 , the mean B-cell count was reduced to 3.4 with mean change of 129.1 from baseline B-cell count. The percentage change from baseline values was $88.5 \%$ and $98.5 \%$ at week 4 and week 24 , respectively, in Rituxirel ${ }^{\mathrm{TM}}$ arm. The baseline mean B-cell count observed for reference arm was 760.1, which showed a decline after the start of treatment with reference arm. At week 24, the mean B-cell count was reduced to 52.5 with mean change of 983.6 from baseline B-cell count. The percentage change from baseline values was $53.0 \%$ and $98.2 \%$ at week 4 and week 24 , respectively, in reference arm. The difference between two treatments for percentage reduction at week 24 was nonsignificant $(P=0.560)$ [Figure 3].

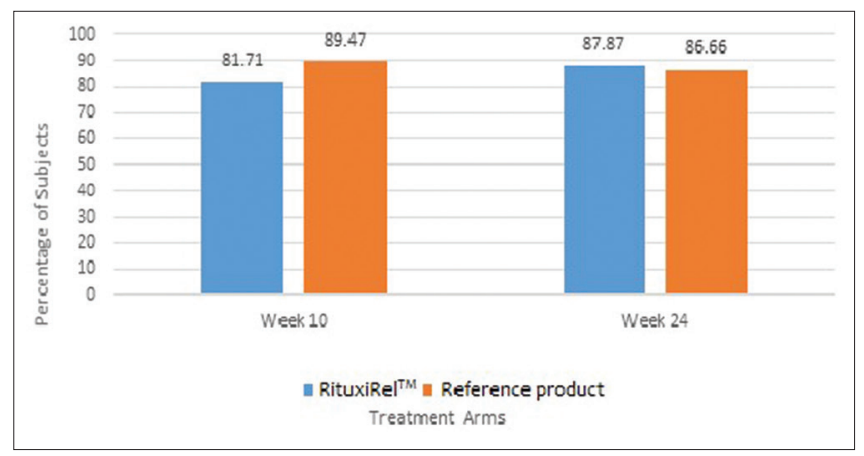

Figure 2: Objective response rate at week 10 and week 24 for Rituxirel ${ }^{\mathrm{TM}}$ and reference arm
Considering biosimilarity guidelines, the observed efficacy results in this study for Rituxirel ${ }^{\mathrm{TM}}$ were comparable for primary efficacy endpoint (ORR at week 24) with reference arm. The pharmacodynamics effect observed with both Rituxirel ${ }^{\mathrm{TM}}$ and reference arm. Both Rituxirel ${ }^{\mathrm{TM}}$ and reference arm were found to be comparable in terms of efficacy and biosimilarity.

\section{Safety results}

In the study, all 105 patients who were dosed were considered for the safety population. AEs were analyzed in the safety population. All AEs were classified according to the Medical Dictionary for Regulatory Activities version 16.0.

In the Rituxirel ${ }^{\mathrm{TM}}$ arm, the most commonly reported TEAEs were related to blood and lymphatic system disorders (45 [52.94\%]) followed by gastrointestinal disorders (43 [50.59\%]), general disorders, and administration site conditions (34 [40.00\%]). In the reference arm, the most commonly reported TEAEs were related to blood and lymphatic system disorders (14 [70\%]) followed by general disorders and administration site conditions (13 [65\%]) and gastrointestinal disorders (12 [60\%]).

Table 1: Demographics and baseline characteristics (intention-to-treat population [ $n=108]$ )

\begin{tabular}{lccc}
\hline Variable & $\begin{array}{c}\text { RituxiRel }^{\mathrm{TM}} \\
(\boldsymbol{n}=\mathbf{8 6})\end{array}$ & $\begin{array}{c}\text { Reference } \\
\text { arm }(\boldsymbol{n}=\mathbf{2 2})\end{array}$ & $\begin{array}{c}\text { Total } \\
(\boldsymbol{n}=\mathbf{1 0 8})\end{array}$ \\
\hline Age (years) & & & \\
$n$ & 86 & 22 & 108 \\
Mean & 47.8 & 52.7 & 48.8 \\
SD & 12.58 & 12.34 & 12.63 \\
Median & 49 & 58 & 50 \\
Range & $21.0-72.0$ & $21.0-65.0$ & $21.0-72.0$ \\
Gender, & & & \\
$n(\%)$ & & $6(27.27)$ & $43(39.81)$ \\
Female & $37(43.02)$ & $16(72.73)$ & $65(60.19)$ \\
$\quad$ Male & $49(56.98)$ & & \\
\hline
\end{tabular}

SD - Standard deviation

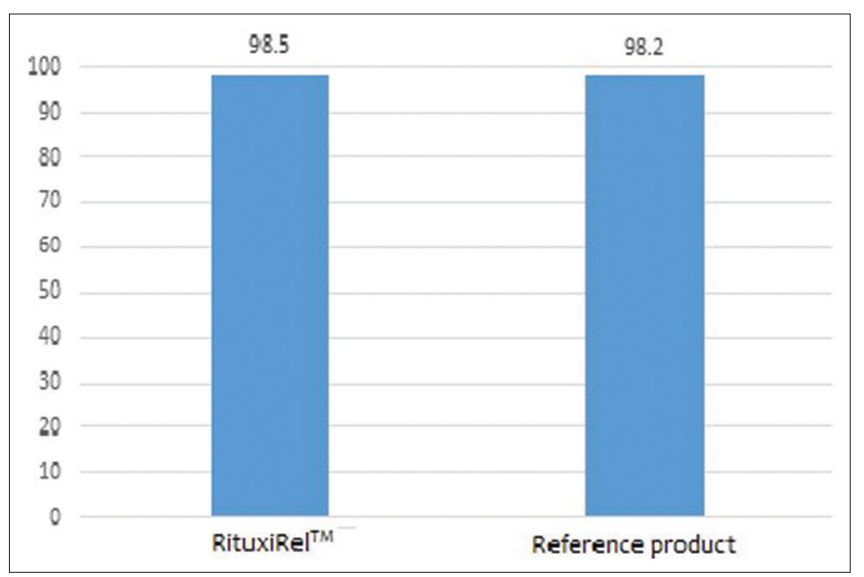

Figure 3: Mean B-cell count for Rituxire ${ }^{\mathrm{TM}}$ and reference arm 
There were $74(87.06 \%)$ patients in the Rituxirel ${ }^{\mathrm{TM}}$ arm and $18(90 \%)$ patients in the reference arm who had at least one $\mathrm{AE}$ in the study. There were 37 (43.53\%) patients in the Rituxirel $^{\mathrm{TM}}$ arm and $8(40 \%)$ patients in the reference arm with at least one SAE in the study. There were a total of 82 SAEs were reported in the study [Figure 4].

Sixty-six SAEs were reported in 37 patients in Rituxirel ${ }^{\mathrm{TM}}$ arm and 16 SAEs were reported in 8 patients in reference arm.

There were 14 deaths reported in this study. Out of 14 deaths, $11(12.94 \%)$ deaths were reported in the Rituxirel ${ }^{\mathrm{TM}}$ arm and $3(15 \%)$ deaths were reported in the reference arm. There were $2(2.35 \%)$ patients from Rituxirel ${ }^{\mathrm{TM}}$ arm and $2(10 \%)$ patients from the reference arm who discontinued the study due to an $\mathrm{AE}$.

Considering the toxicity profile of R-CHOP, population under study, type of tumor, stage of the disease, other age-associated complications, the observed severe and fatal cases reported in this study were comparable in both groups and consistent with the known safety profile observed with R-CHOP therapy.

The most commonly reported AEs were similar in both treatment arms. The frequency and severity of AEs were comparable for both and reference arm. Patients were with at least one TEAE, 74 (87.06\%) patients in the Rituxirel ${ }^{\mathrm{TM}}$ arm and $18(90.00 \%)$ patients in the reference arm.

The SAEs reported in both Rituxirel ${ }^{\mathrm{TM}}$ and the reference arm were similar (37 [43.53\%] patients in the Rituxirel ${ }^{\mathrm{TM}}$ arm and $8[40 \%]$ in reference arm).

No major safety-related new concerns were noted with investigations such as electrocardiography, lactate dehydrogenase, or hematology in treatment arms. No statistical significant difference was observed between both the treatment arms for causality, number of AEs, TEAEs, severe and serious TEAEs.

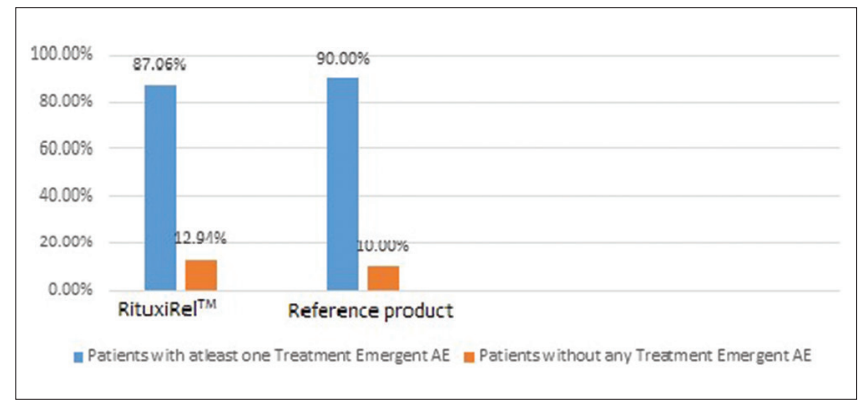

Figure 4: Treatment-emergent adverse events

\section{DISCUSSION}

DLBCL is the most common subtype of non-Hodgkin lymphoma accounting for $30 \%-40 \%$ of all cases, whereas FL is the most common subtype of low-grade (indolent) lymphoma, making up $20 \%-30 \%$ of all non-Hodgkin lymphomas.

Rituximab is the first monoclonal antibody to have been registered for the treatment of B-cell lymphomas and has been extensively evaluated in DLBCL and FL either as combination or monotherapy.

DLBCL advances very quickly, it usually requires immediate treatment. Adding rituximab to $\mathrm{CHOP}$ results in an increase in long-term survival of almost $20 \%$. A 6-year event-free survival was $55.8 \%$ for patients assigned to chemotherapy alone and $74.3 \%$ for those assigned to chemotherapy plus rituximab. ${ }^{15]}$

In FL, rituximab as a single agent has been compared with watchful waiting in patients with low disease burden and has been found to be associated with improved progression-free survival as well as improved quality of life. In patients with a greater burden of disease, chemotherapy is commonly given in combination with rituximab Single-agent rituximab in treatment-naive patients with FL has yielded an ORR of $72 \%-73 \%$, with a median time to disease progression of just over 2 years. In the relapsed setting, rituximab has yielded an ORR of $40 \%$, with a median time to disease progression of about 18 months.

Rituxirel $^{\mathrm{TM}}$ arm and reference arm given for six cycles to elderly patients with newly diagnosed DLBCL or FL showed comparable efficacy at week 24 .

The analysis of primary efficacy endpoint, i.e., ORR at week 24 shows comparable response for both Rituxirel ${ }^{\mathrm{TM}}$ arm and reference arm (70.73\% vs. $68.42 \%)$ as a composite for DLBCL and FL. The ORR was observed to be $81.71 \%$ in the Rituxirel ${ }^{\mathrm{TM}}$ arm compared to $89.47 \%$ patients in reference arm at week 10 .

In the Rituxirel ${ }^{\mathrm{TM}}$ arm, the most commonly reported TEAEs were related to blood and lymphatic system disorders (45 [52.94\%]) followed by gastrointestinal disorders (43 [50.59\%]), general disorders, and administration site conditions $(34[40 \%])$. In the reference arm, the most commonly reported TEAEs were related to blood and lymphatic system disorders (14 [70\%]) followed by general disorders and administration site conditions (13 [65.00\%]) and gastrointestinal disorders (12 [60.00\%]). 
There were 74 (87.06\%) patients in the Rituxirel ${ }^{\mathrm{TM}}$ arm and $18(90 \%)$ patients in the reference arm who had at least one $\mathrm{AE}$ in the study.

\section{CONCLUSION}

CHOP combined with rituximab was highly effective in untreated patients with indolent B-NHL, especially FL, either in a concurrent or sequential combination, with acceptable toxicities. Based on the efficacy and safety results at week 24, Rituxirel ${ }^{\mathrm{TM}}$ arm was found to be effective and safe as reference arm. Rituxirel ${ }^{\mathrm{TM}}$ can be a suitable treatment option in patients with NHL based on the current study conducted with this new biosimilar.

\section{Acknowledgment}

We acknowledge the following investigators who participated in the study and were instrumental in conducting and completion of the trial successfully at their respective centers to generate this data (Asha Anand, Ashish Kaushal, Ashok Kumar Diwan, Cecil Ross, Chetan Deshmukh, J. B. Sharma, Kirushna Kumar, Krishna Prasad, Minish Jain, Murali Krishna Voonna, Niraj Bhatt, Rajendersingh Arora, R. K. Grover, Sankara Mahadev, Shekhar Patil, Sujata Vasani,
Sulabhchandra Bhamare, Surender Beniwal, and Unmesh Takalkar).

Financial support and sponsorship

This study was supported in the form of financial grant and provided the investigational product and innovator drug.

\section{Conflicts of interest}

This is a regulatory multicentric phase III clinical study with the regulatory approval in India conducted by Reliance Life Sciences Pvt. Ltd. The authors declare that there is no conflict of interest.

\section{REFERENCES}

1. Alizadeh AA, Eisen MB, Davis RE, Ma C, Lossos IS, Rosenwald A, et al. Distinct types of diffuse large B-cell lymphoma identified by gene expression profiling. Nature 2000;403:503-11.

2. Vallabhajosyula S, Baijal G, Vadhiraja BM, Fernandes DJ, Vidyasagar MS. Non-Hodgkin's lymphoma: Is India ready to incorporate recent advances in day to day practice? J Cancer Res Ther 2010;6:36-40.

3. Castillo J, Winer E, Quesenberry P. Newer monoclonal antibodies for hematological malignancies. Exp Hematol 2008;36:755-68.

4. RituxiRelTM Clinical Study Report, Version 2.0 Dated 2015; Reliance Life Sciences Pvt. Ltd., India.

5. Hauptrock B, Hess G. Rituximab in the treatment of non-Hodgkin's lymphoma. Biologics 2008;2:619-33. 